\title{
A Development of Automotive Recognition Street Light Control with Sound Perception Technology
}

\author{
Wonchul Choi ${ }^{1}$ and Choongchae Woo ${ }^{2}$ \\ ${ }^{1}$ Dept. of Electronic Engineering, Hanseo University, Hanseo 1 Ro $46 \mathrm{Haemi}$ \\ Seosan Chungnam South Korea,356-706 \\ ${ }^{2}$ Dept. of Electronic Engineering, Hanseo University, Hanseo 1 Ro 46 Haemi \\ Seosan Chungnam South Korea,356-706 \\ 1.comling2497@gmail.com,2woo9@hanseo.ac.kr
}

\begin{abstract}
In this paper, proposed a new lighting control system which can reduce power consumption compared to conventional street lamps and intelligently control the light efficiently depending on whether there is a vehicle on the street. The new lighting control system proposed by this paper detects the presence of cars by collecting and analyzing sounds generated by the movement of cars. Then, the system controls lighting of street lamps based on the above car detection information, and turns on the street lamps sequentially by transmitting the car detection information. Experimental results showed that lightings were controlled based on the presence of cars and that operations of the lamps were made by turning on the lights sequentially by determining the moving direction of cars. This system is considered a technology that can reduce energies by applying to local roads with a few cars moving or national highways where lights are always turned on with low energy efficiency.
\end{abstract}

Keywords: automotive recognition, lighting control, sound recognition, streetlight

\section{Introduction}

Countries all over the world are carrying out various energy policies to cope with the energy resource problem. Korean Government has also presented 3 strategies and 10 policy directions under the vision of 'Low Carbon, Green Growth' to reduce the energy consumption [1-3]. To be in step such trend, there are many ongoing studies of lighting control technologies to reduce the energy by better managing the streetlight which is one of examples of excessive energy consumption as it is always lighted regardless of existence of traffic on the road.

Under the conventional streetlight control system, a streetlight controller installed in each street lamp receives the control signal through the PLC (Power Line Communication) or RF (Radio Frequency) communication method from a distribution box to control turning on/off of the street lamp; monitors the short circuit, power consumption and possible failure and sends the data to the distribution box [1-6].

Such system of controlling the streetlight through the distribution box is applied to most street lamps, and such system is an example of energy waste as the street lamps are turned on or off only by time regardless of brightness of the road.

Although the illumination of the road is measured using an optical sensor to such problem, the sensor has the problem of creating error caused by foreign substances such as snow or dust as time passes. A more advanced system detects the vehicle on the road with an IR sensor, but the system turns on the street lamp when it detects a vehicle, and thus it is an inefficient system for securing visibility which is the main purpose of streetlight [7]. 
In this paper, we propose and develop an advanced streetlight management system which uses a sound recognition function to detect the sound generated when a vehicle moves and turn on a street lamp then sequentially turns on the next street lamp in the direction of the vehicle movement.

The rest of this paper is organized as follows. In Section 2, we introduce the organization of the developed system and computer simulation test. Section 3 describes the theory applied to the developed system and design of the system. Section 4 shows the result of test. Finally, we present the conclusion in Section 5.

\section{System Organization}

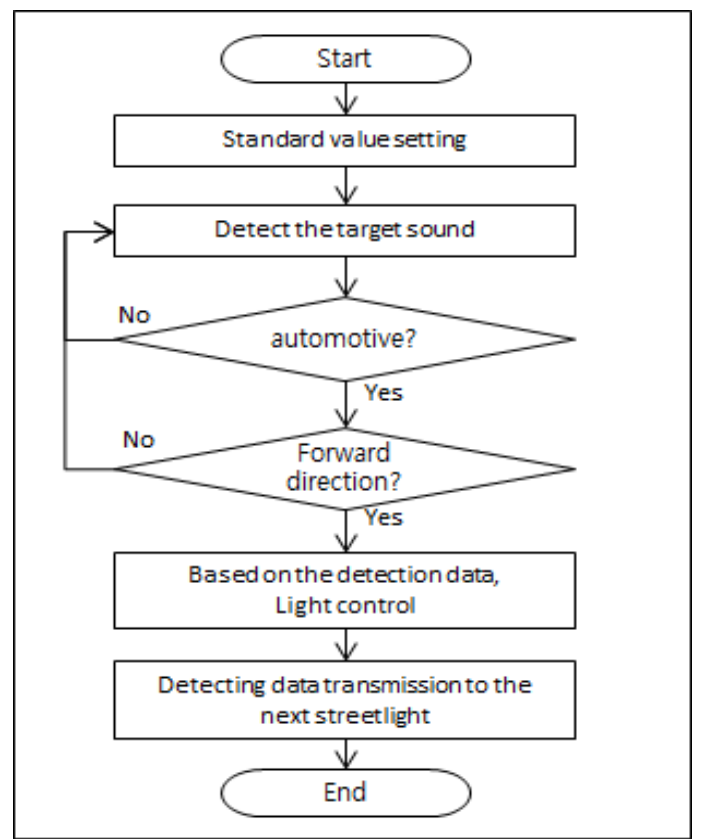

Figure 1. System Flowchart

The developed system consists of a detection unit to detect the sound generated by a moving vehicle, an integrated controller to analyze the existence of a vehicle and its moving direction based on the data from the detection unit, a lighting control unit to control lighting according to the control signal from the integrated controller, and a wireless communication unit to send the vehicle detection data to the next street lamp in the vehicle moving direction. The developed system can detect the vehicle on the road and its moving direction and control the streetlight to reduce the energy consumed by the street lamps.

Figure 1 shows the work flow of the developed system. The developed system detects a sound and compares the acquired sound data with the predefined reference data to determine if the sound is generated by a moving vehicle. It also analyze the acquired sound data to determine the moving direction and send the data to the next street lamp in the vehicle moving direction to control it. To correctly extract the sound generated by a moving vehicle, the sound generated by moving vehicle as well as the noise on the road such as wind sound, sound of rain, sound of wind and human voice were also collected. The standardization size and frequency of the sounds were analyzed through a computer simulation test. 


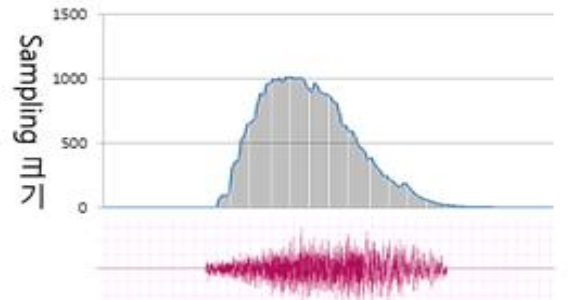

Automotive

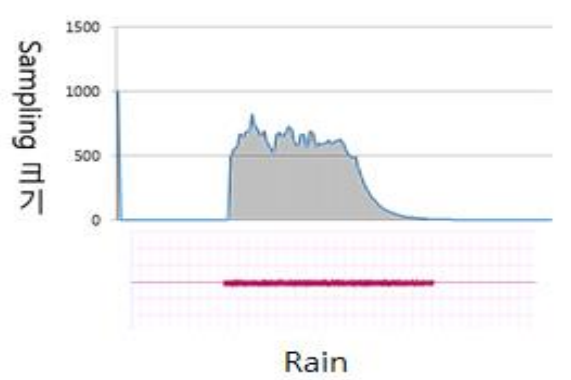

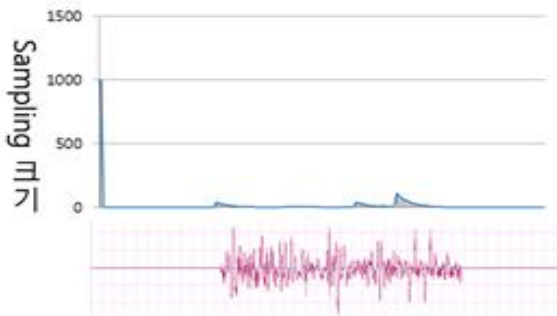

Wind

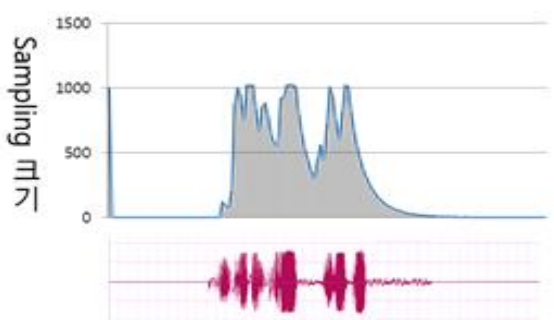

Human Voice

Figure 2. Sound Sampling Data

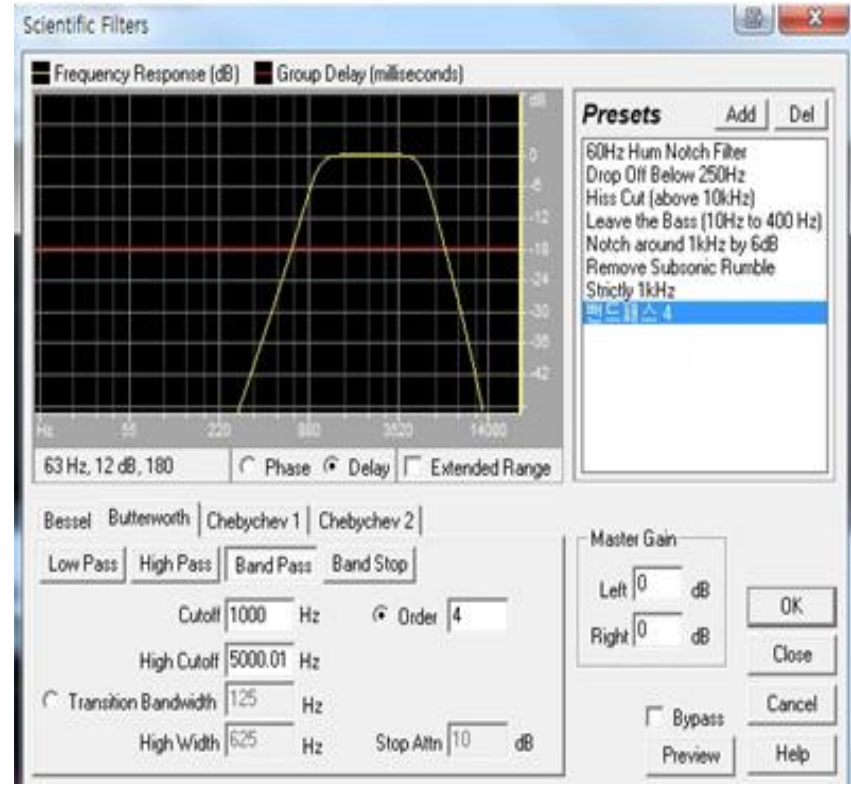

Figure 3. BPF Implemented Through Simulation

Figure 2 shows the standardization size and spectrum data of the actual sound of a vehicle moving on a road as well as the noise on the road such as wind sound, sound of rain, sound of wind and human voice. The analysis of the standardization size and frequency of the acquired data with Cool edit pro (simulation program) by Syntrillium Software indicated that application of a band-pass filter which only passes the frequency of $1 \mathrm{kHz} \sim 5 \mathrm{kHz}$ can minimize the noise when extracting the vehicle sound. Figure 3 shows the band-pass filter developed with the simulation program. 


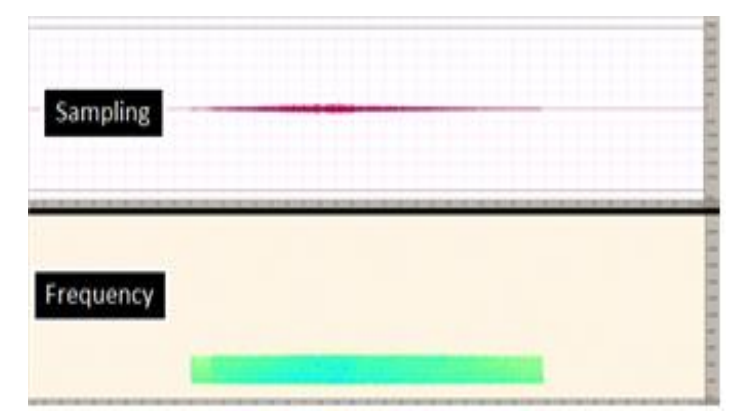

Figure 4. The Automotive Sound Data Applying the Bandpass Filter

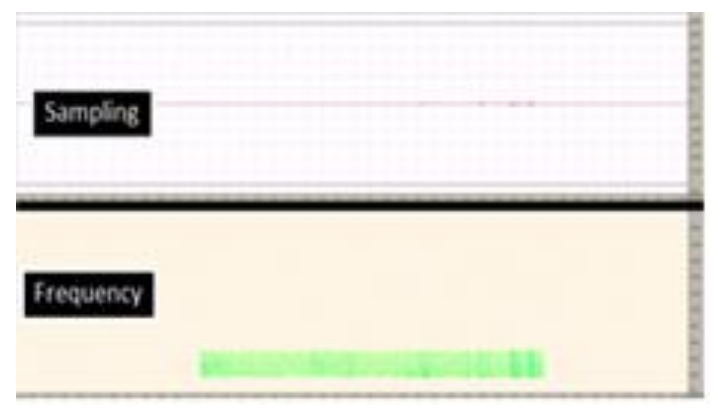

Figure 5. The Wind Sound Data Applying the Bandpass Filter

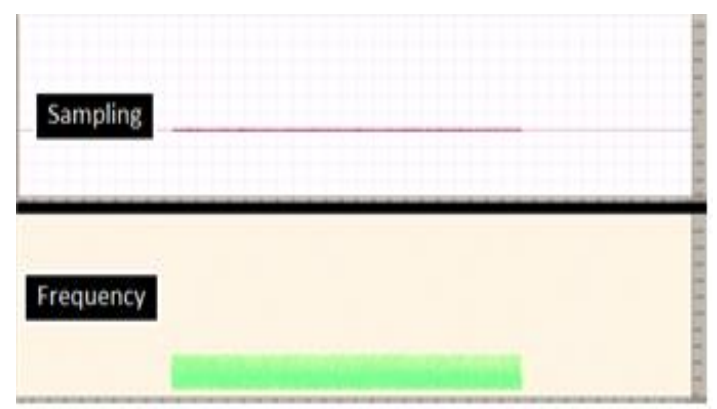

Figure 6. The Rain Sound Data Applying the Bandpass Filter

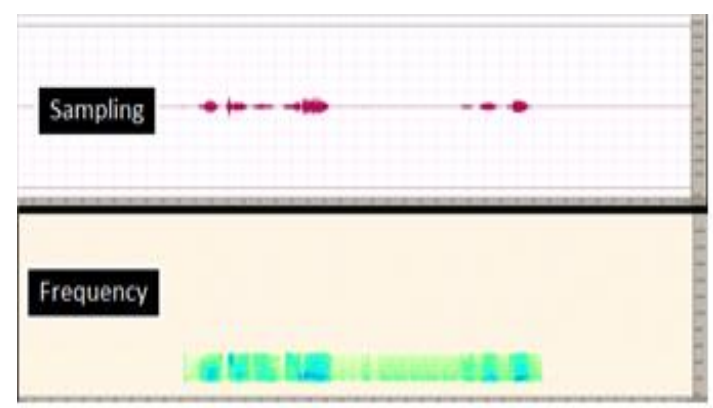

Figure 7. The Voice Sound Data Applying the Bandpass 
Applying of the developed band-pass filter to the acquired sound showed that the sound of rain and wind could be clearly distinguished from the sound of vehicle but it was difficult to separate the human voice from vehicle sound.

Figure 4 shows the standardization size and spectrum data as the result of band-pass filter applied to the sound generated by the vehicle moving on a road.

Figure 5 and 6 show the standardization size and spectrum data as the result of bandpass filter applied to the sound of wind and the sound of rain, respectively.

Figure 7 shows the standardization size and spectrum data as the result of band-pass filter applied to the human voice.

Analysis of the above data concluded that the noise problem could be solved if the system assumed the sound came from a moving vehicle if a certain level or high is maintain a specific period and assumed the sound was a noise and removed the noise if the condition was not satisfied.

\section{System Design}
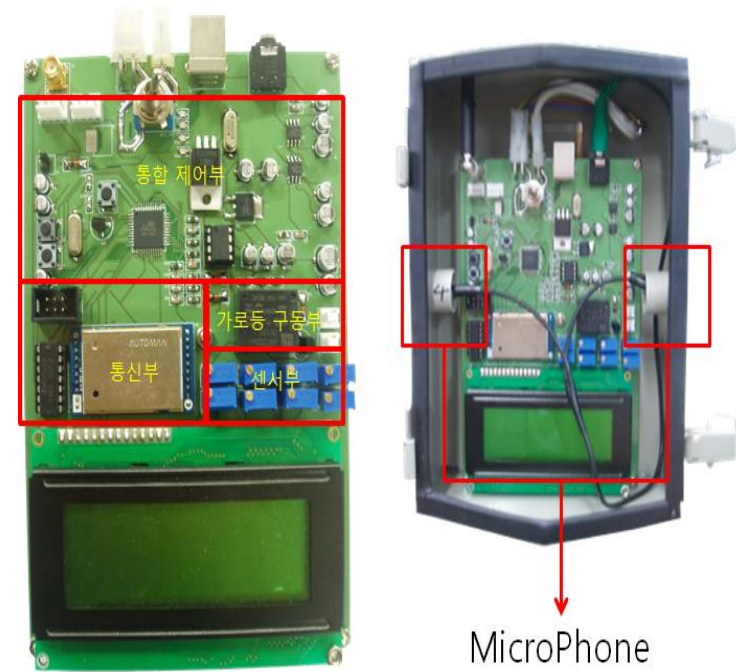

Figure 8. System Board

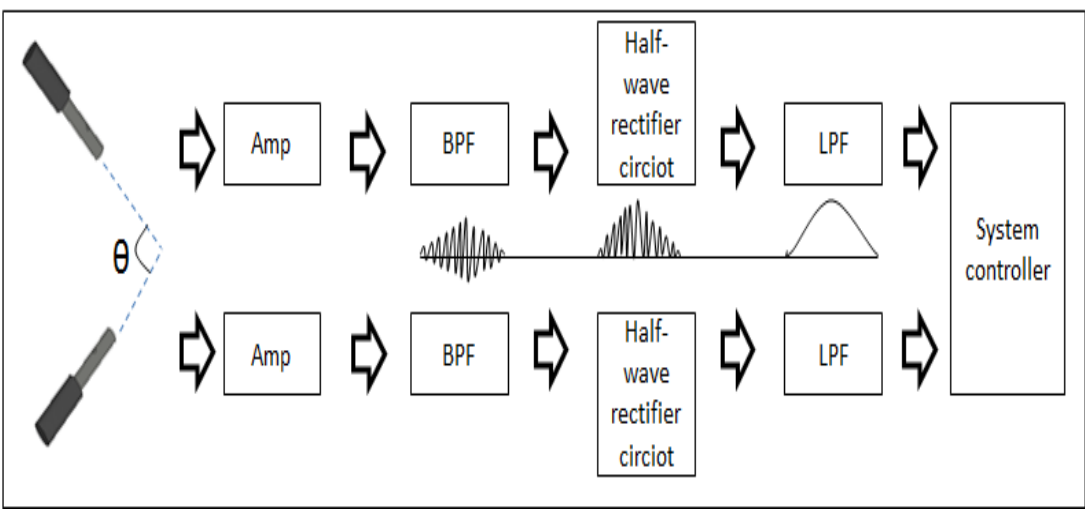

Figure 9. Automotive Sound Retrieving System Using Sensor 


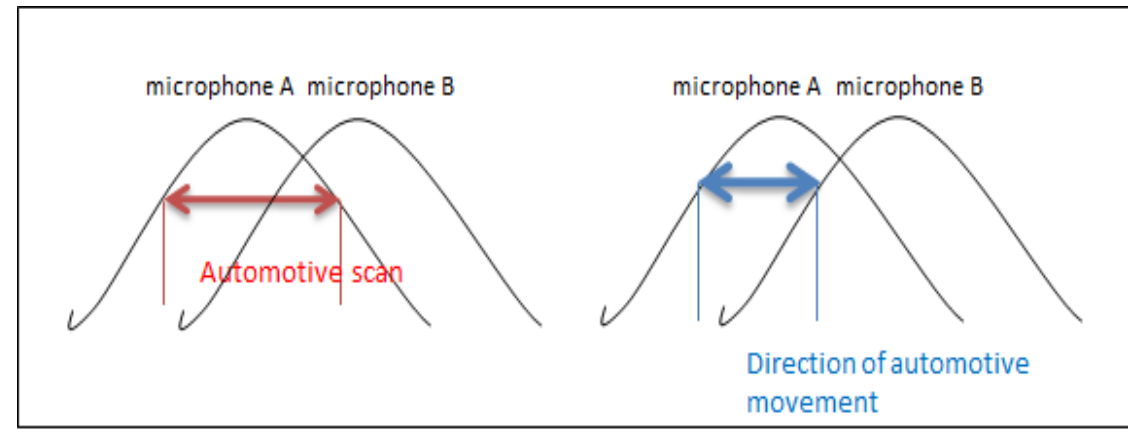

\section{Figure 10. Automotive Scan and Direction of Automotive Movement Detection Method}

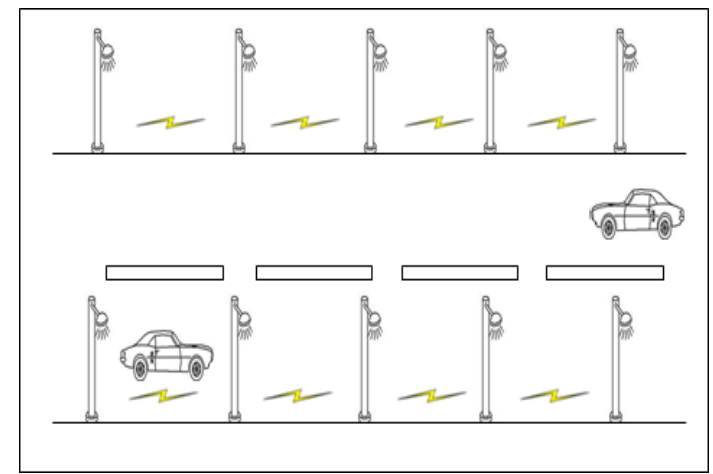

Figure 11. Information Sharing Of Wireless Communication between Streetlight

Based on the conclusion from computer simulation and theoretical background, the detection unit, communication unit, control unit, and streetlight operation unit were developed as shown in Figure 8.

Figure 9 shows the work flow of the sensor unit operation to detect a moving vehicle and its direction. The sensor unit acquires and amplifies the sound signal from two microphones installed in a specific angle and extracts only the sound signal from a moving vehicle by applying the band-pass filter to the amplified sound signal. The extracted vehicle sound signals are flattened by the half-wave rectifier circuit and lowpass filter for easy sampling and sent to the control unit.

Figure 10 shows how the vehicle and its moving direction are detected with the signal input to the sensor unit. The vehicle sound is identified when sound signal collected from an installed microphone remains above a specific level for a specific period, and the moving direction of a vehicle is determined by comparing the sound collected earlier and the sound collected later from the sounds collected by two microphones installed in a specific angle. The control unit recognizes a vehicle if the received signal remains above a preset level or above for a specific period or longer and checks the time difference between signals collected from two microphones to determine the vehicle moving direction.

Figure 11 shows an example of sharing of the data of vehicle detection and moving direction between the street lamps. The communication unit used an RF communication module using the $400 \mathrm{MHz}$ band frequency to minimize the interference and secure the data transfer distance. The wireless network was built in order to transmit the vehicle recognition data and turn on the next street lamp before the vehicle reached it. 


\section{Result}

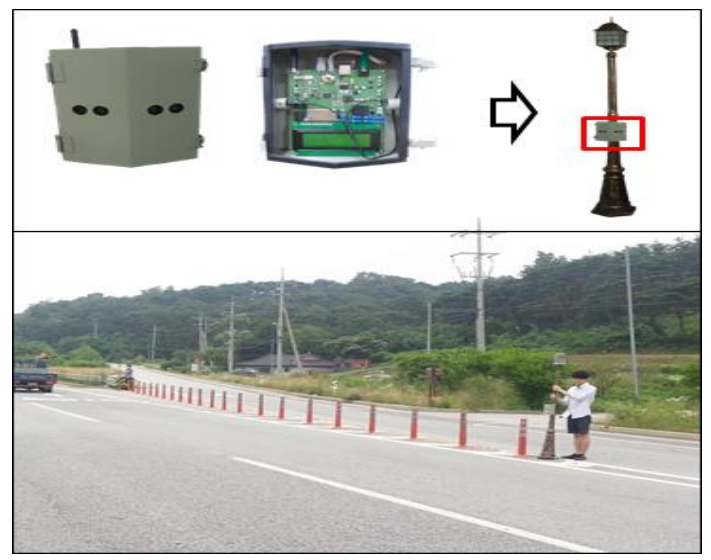

Figure 12. Field Test Methods

The developed system was designed for a microphone installed on both sides of the case to determine the existence of a vehicle and its moving distance. Because of limited time and cost, the developed system was not tested with actual street lamps but installed on the model street lamps in similar environment as shown in Figure 12 and appled on an actual road. Its operation was checked when a vehicle moves, and the sound data were acquired at the same time to check if the fabricated system operates normally.

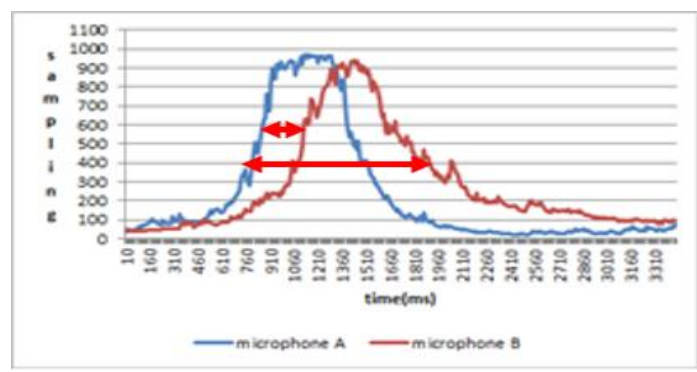

Figure 13. One Automotive Forward Direction

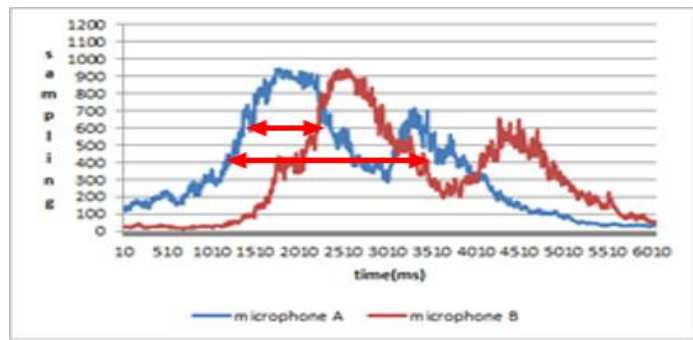

Figure 15. Two Automotive Forward Direction

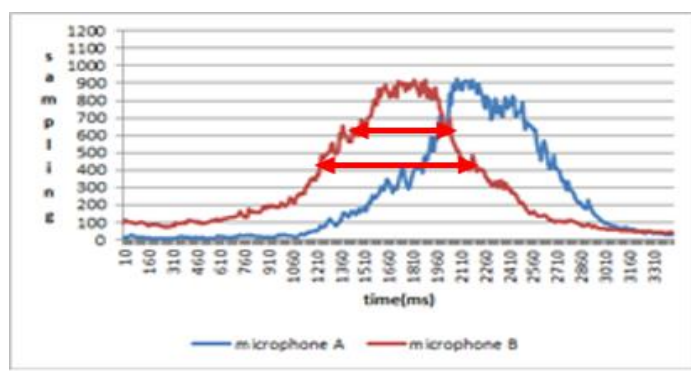

Figure 14. One Automotive Reverse Direction

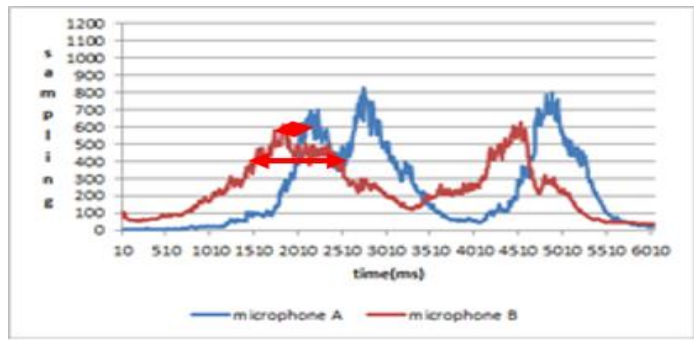

Figure 16. Two Automotive Reverse Direction

Figure 13 and 14 show the data sampling the size of the sound generated when a vehicle moved forward and backward on an actual road with $10 \mathrm{~ms}$ time delay.

Figure 15 and 16 show the data sampling the size of the sound generated when two vehicles moved forward and backward on an actual road with 10ms time delay. 


\section{Conclusion}

The sound recognizing streetlight control system described in this paper has following benefits. First, it not only reduces the energy but also saves the cost by intelligently controlling the street lamps which normally remains lighted continuously. Second, the applied sound recognition has less risk of error than the conventional system of turning on a street lamp by measuring the brightness. Third, sharing of control data among the street lamps enables efficient management of many street lamps and efficient control of each street lamp. Fourth, the system can be easily installed in the existing street lamps thus can reduce the initial replacement cost or installation cost.

As such, the sound recognizing streetlight control system proposed in this paper can be applied to the street lamps which are always lighted inefficiently on the remote roads where there is relatively less traffic and efficiently save the energy.

\section{References}

[1] W. Choi and C. Woo, "A development of Automotive recognition streetlight lighting control with sound recognition technology", Advanced Science and Technology Letters, vol. 86, no. 2, (2015), pp. 58-61.

[2] J. Lee, K. Nam, S. Jeong, S. Choi, H. Ryoo and D. Kim, "Design of Zigbee based Street Light Control System", Journal of The Korean Institute of Electrical Engineers(KIEE), (2007), pp. 935-936.

[3] C. M. De Dominicis, A. Flammini, E. Sisinni, L. Fasanotti and F. Floreani, "On the development of a wireless self-localizing streetlight monitoring system", 2011 IEEE Sensor Applications Symposium(SAS), (2011), pp. 233-238.

[4] J. Liu, C. Feng, X. Suo and A. Yun, "Street Lamp Control System Based on Power Carrier Wave", 2008 Intelligent Information Technology Application Workshops (IITAW), (2008), pp. 184-188.

[5] K. Lee and S. Lee, "Implementation of an Effective Streetlamp Panel Board Controller", Journal of The Korean Institute of Information Technology, vol. 10, no. 4, (2012), pp. 43-48.

[6] C. In, D. Yoon and C. Lin, "New Lighting Control System for Light Devices", Journal of IKEEE, vol. 15C, no. 4, (2011), pp. 261-266.

[7] J. Kim, D. Lee, TengYuzhao, D. Jeong and D. Song, "A Light Lamp Control System for Saving Energy", Journal of The Korean Institute of Information Technology, (2009), 930-933.

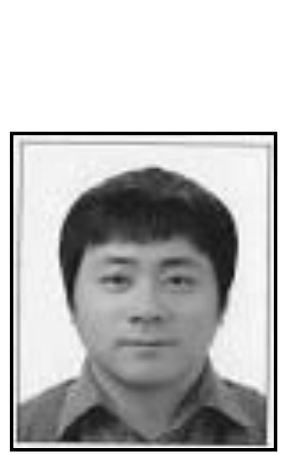

\section{Authors}

Wonchul Choi received the B.S. degrees in Electronic engineering at Hanseo University, Seosan, Korea in 2011, and M.S. degrees in Electronic Engineering at Hanseo University, Seosan, Korea in 2014, His current research interests include the embedded system and micro controller unit.

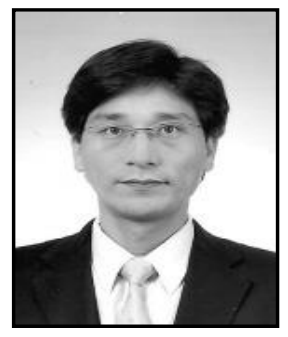

Choongchae Woo received the B.S. degrees in Electronic engineering at Sunchon National University, Sunchon, Korea in 2000, and M.S. and Ph.D. degrees in Electrical and Electronic Engineering at Yonsei University, Seoul, Korea in 2002, and 2007, respectively. From September 2007 to February 2009, he was a senior engineer in Samsung Electronics, Suwon, Korea where he developed Mobile WiMAX systems for broadband wireless services. In March 2009, he joined the Department of Electronics Engineering at the Hanseo University as an Assistant Professor. His current research interests include the field of wireless communications focusing on multicarrier systems and resource allocation algorithms. 